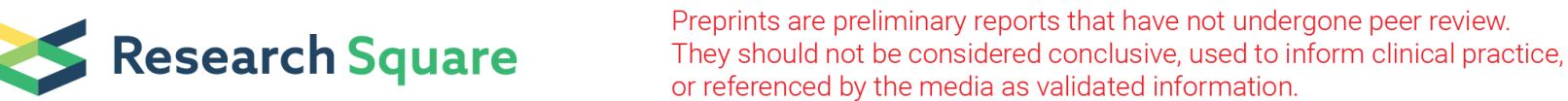

\section{Early Risk Prediction of BCR-ABL Kinase Domain Acquired Mutations in Philadelphia Chromosome Positive Acute Lymphoblastic Leukemia}

\section{Rongrong Chen}

Zhejiang University School of Medicine First Affiliated Hospital

\section{Lulu Wang}

Zhejiang University School of Medicine First Affiliated Hospital

\section{Ting Shi}

Zhejiang University School of Medicine First Affiliated Hospital

\section{Lixia Zhu}

Zhejiang University School of Medicine First Affiliated Hospital

Xiujin Ye ( $\nabla$ yxjsunny@zju.edu.cn )

Zhejiang University School of Medicine First Affiliated Hospital https://orcid.org/0000-0002-4553-2751

\section{Primary research}

Keywords: acute lymphoblastic leukemia, TKI resistance, BCR-ABL kinase domain mutations

Posted Date: February 4th, 2021

DOI: https://doi.org/10.21203/rs.3.rs-170587/v1

License: (c) (1) This work is licensed under a Creative Commons Attribution 4.0 International License. Read Full License 


\section{Abstract}

Tyrosine kinase inhibitors (TKI) resistance is a predominant cause of therapy failure of Philadelphia chromosome-positive acute lymphoblastic leukemia (Ph+ ALL). The mutation in the BCR-ABL kinase domain, as the indicator of drug resistance, suggested recurrence and poor prognosis. Our data aimed to evaluate the factors having significant roles in the prognostic value of the acquired BCR-ABL mutations. Three hundred and thirteen Ph+ ALL patients (64 patients with BCR-ABL mutations and 171 patients without BCR-ABL mutations) were enrolled in this study. Univariate and multivariable logistic regression analysis was used to evaluate the relationship between clinical features and BCR-ABL KD mutation. Univariate analysis showed that the patients with higher WBC $(P=0.005)$, MMR after first induced chemotherapy $(P=0.053)$, and Age $\geq 45$ years $(P=0.072)$ were more likely to occur BCR-ABL mutations during the treatments. Finally, multivariable logistic regression indicated that higher WBC $(\geq 22 \times 109 / L)$ was an independent risk of resistance to TKI and raise the possibility of KD mutations in Ph+ ALL patients. The happen of BCR-ABL mutations mean poor prognosis with shorter OS $(P=0.000)$ and allogeneic hematopoietic stem-cell transplantation (allo-HSCT) would improve the long-term survival (OS: $P=0.000$ ). Conversely, for the defined low-risk populations, no significant difference was found between the transplant group and the non-transplant group in subgroup analysis, providing a rationale to potentially avoid allo-HSCT in this subgroup of patients.

\section{Introduction}

The prognosis of Philadelphia chromosome-positive acute lymphoblastic leukemia $(\mathrm{Ph}+\mathrm{ALL})$ has been substantially improved since tyrosine kinase inhibitors (TKIs) was used as a frontline treatment. Combined chemotherapy with TKI increased Ph + ALL's 5-year overall survival rate from 10-40\% 50\% and the hematologic CR rate exceeds $90 \% .{ }^{[1-3]}$. Despite the clinical improvement of TKI treatment in $\mathrm{Ph}$ $+A L L$, there are quite a number of patients experiencing Tyrosine kinase inhibitors drug resistance caused by the acquisition of secondary mutations in the BCR/ABL kinase domain $(K D)^{[4]}$. The acquired gene alteration changes the conformation of BCR-ABL and inhibits the anti-tumor effect of TKI through influencing its binding to TKI. Previous studies have shown that BCR-ABL could be detected in almost $70 \%-80 \% \mathrm{Ph}+\mathrm{ALL}$ patients who own drug resistance ${ }^{[5]}$. MRD monitoring and BCR-ABL KD mutations were generally essential for the successful management of $T K I$ in $P h+A L L{ }^{[2,6]}$. Except for the discontinuation of TKI treatment, the degree of genetic instability, and additional cytogenetic abnormalities (ACAs) may foster the acquisition of BCR-ABL KD mutations in ALL ${ }^{[7,8]}$.

For the populations who are more likely to occur drug resistance mutations, the early choice and management of TKI are particularly important. The identification of predictive factors for patient TKI resistance stratification is therefore of crucial importance in management. However, few studies have focused on the early predictors of BCR-ABL mutations in Ph + ALL. Here, we explored the predictive value of the disease features and the early molecular response in $\mathrm{Ph}+\mathrm{ALL}$. Meanwhile, further analysis of survival also is performed which may guide the treatment strategies in different mutation-risk subgroups. 


\section{Method}

\section{Patients and data collection}

From January 2010 to December 2019, 313 patients with Philadelphia chromosome (Ph)-positive acute lymphoblastic leukemia (ALL) who presented to the First Affiliated Hospital, Zhejiang University, were systematically analyzed. Patients were excluded from the study if their duration of TKI or chemotherapy was less than 3 months or they used TKI irregularly. Finally, a total of 235 patients were involved in the study, and according to whether mutations were happening in BCR/ABL kinase domain (KD), patients were divided into two groups (patients with BCR-ABL mutations $n=64$ and patients without BCR-ABL mutations $n=171$ ) See Fig. 1 for details. The diagnosis of ALL was based on the criteria of the 2018 World Health Organization (WHO). The Philadelphia chromosome $t(9 ; 22)(q 34 ; q 11)$ or the BCR-ABL fusion gene was detected by conventional cytogenetic analysis. ACAs were defined as additional cytogenetic abnormalities except for the Philadelphia chromosome. The P190 or the P210 BCR-ABL fusion protein variants were detected by PCR analyses.

\section{MRD monitoring and gene mutations assessment (Direct sequencing)}

The Monitoring of morphology and MRD used bone marrow (BM) samples. Analysis of MRD based on $B C R-A B L$ was performed in this research's reference laboratory using a quantitative real-time polymerase chain reaction (QT-PCR) and estimating the BCR-ABL1 copy numbers to quantify the residual disease burden in Ph + ALL patients based on Pfeifer's research ${ }^{[9]}$. MRD was monitored after the first induction of chemotherapy and subsequent chemotherapy evaluations. Direct sequencing was performed to identify mutations in $B C R / A B L$ kinase domain in patients at the onset or progression of the disease.

\section{Therapeutic regimen}

Imatinib was the first treatment option for $\mathrm{Ph}+\mathrm{ALL}$ patients in our study while dasatinib and ponatinib would be considered in patients with imatinib resistance or at high risk or prone to bone marrow suppression. The VICP regimen as the background induction treatment (vindesine $4 \mathrm{mg}$ i.v. on days 1, 8 , 15; idarubicin $45 \mathrm{mg} / \mathrm{m} 2$ i.v. on days 1 ; cyclophosphamide $600 \mathrm{mg} / \mathrm{m} 2$ on day 1; dexamethasone $10 \mathrm{mg} /$ day on days 1 to 14) was the most universal treatment used in our department. Initial imatinib doses were $400 \mathrm{mg}$ per day $(n=194)$ and dasatinib doses were $100 \mathrm{mg}$ per day $(n=41)$. The administration of TKIs was based on molecular monitoring of BCR/ABL and BCR/ABL mutations: the same TKIs drug previously used was administered as preemptive treatment in the cases without mutations; sensitive TKIs were used in the patients with mutations. Allo-HSCT had been performed in 5 (7.8\%) and $52(30.4 \%)$ patients, respectively.

\section{Definitions of treatment response and end points}

Marrow CR was defined as no more than $5 \%$ of lymphoblasts found with bone marrow morphology. A relative $B C R / A B L$ quantification of less than $0.1 \%$ was defined as major molecular response (MMR). 
Overall survival (OS) time was defined as the time from the clear diagnosis of the patient's disease until death (including the death of any cause) or missing or the end of follow-up. Disease-free survival (DFS) refers to the interval from the date of $\mathrm{CR}$ to the date of leukemia relapse, death (death from any cause) or follow-up deadline.

\section{Statistical analysis}

SPSS 19.0 software and Stata were used for statistical analysis. Measurement data were compared using the independent-samples T-test. Count data were compared using the chi-square test or Fisher exact test. Survival analysis using Kaplan-Meier method and the curves were performed using GraphPad Prism. Univariate logistic regression analysis was used to evaluate the relationship between clinical features and BCR-ABL KD mutation. We classified the variables which could associated with BCR-ABL mutation according to the median, and included these classified variables in further multivariate logistic regression analyses. The criterion for statistical significance was $p<0.05$.

\section{Result}

\section{Patient characteristics}

Among the 235 patients with $\mathrm{Ph}+\mathrm{ALL}$ in the hematology department during the study period, there were 64 cases in the patients with BCR-ABL mutations group (35 males and 29 females) and 171 cases in the patients without BCR-ABL mutations group (71 males and 100 females). There were relatively higher WBC and LDH in patients with BCR-ABL mutations group $(P=0.01, P=0.004$ and $P=0.007$, respectively) when compared with the patients without BCR-ABL mutations group. There were no significant differences in the other variables between the two categories $(P>0.05)$. See Table 1 for details 
Table 1

The clinical characteristics of $\mathrm{Ph}+\mathrm{ALL}$ patients with $\mathrm{BCR}-\mathrm{ABL}$ mutation or without BCR-ABL mutation

$\begin{array}{ll}\begin{array}{l}\text { Patients with BCR-ABL } \\ \text { mutations }\end{array} & \begin{array}{l}\text { Patients without BCR- } \\ \text { ABL mutations }\end{array} \\ (n=64) & (n=171)\end{array}$

P-value

\begin{tabular}{|l}
\hline Gender (male/female, cases) \\
\hline Age [years, M(range)] \\
WBC [×109/L, M(range)] \\
Hemoglobin [g/L, M(range)] \\
\hline Platelets [×109/L, M(range)] \\
Lactate dehydrogenase [U/L, \\
M(range)] \\
\hline $\begin{array}{l}\text { Bone marrow blast percentage [\%, } \\
\text { M(range)] }\end{array}$ \\
$\begin{array}{l}\text { Relative BCR/ABL quantification [\%, } \\
\text { M(range)] }\end{array}$ \\
MMR after first induced \\
chemotherapy [\%, M(range)]
\end{tabular}

Yes
No

$35 / 29$

$51(18-76)$

$71 / 100$

0.113

44.8(1-403)

$43(17-72)$

0.077

103(36-643)

16.2(2-403)

$0.004^{*}$

38.5(6-190)

97(40-166)

0.143

792(188-7638)

36(2-580)

0.427

526(136-7209)

0.007 *

82.5(38-96)

85(25-98)

0.938

49.5(1-109)

55(1-195)

0.970

(range)]

0.051

17(26.6)

69(40.4)

47(73.4)

102(59.6)

$\mathrm{BCR} / \mathrm{ABL}$ subscript type [cases (\%)]

0.473

P190

44(68.8)

109(63.7)

P210

20(31.1)

62(36.6)

WT1 [cases (\%)]

0.665

$\geq 0.5 \%$

9(14.1)

28(16.4)

$\bowtie 0.5 \%$

55(85.9)

143(83.6)

Additional cytogenetic abnormalities [cases (\%)]

$\begin{array}{lll}\text { Yes } & 17(26.6) & 43(25.1) \\ \text { No } & 34(53.1) & 94(55) \\ \text { Unknown } & 13(20.3) & 34(19.9)\end{array}$

TKI types used at diagnosis

0.108

* $P<0.05$ was considered statistically significant. 


\begin{tabular}{|lll|}
\hline & $\begin{array}{l}\text { Patients with BCR-ABL } \\
\text { mutations } \\
(\mathbf{n}=64)\end{array}$ & $\begin{array}{l}\text { Patients without BCR- } \\
\text { ABL mutations } \\
(\mathbf{n}=171)\end{array}$ \\
\hline Imatinib & $57(89.1)$ & $137(80.1)$ \\
\hline Dasatinib & $7(10.9)$ & $34(19.9)$ \\
\hline$* P<0.05$ was considered statistically significant. & \\
\hline
\end{tabular}

Among the 235 patients, 219 (93.2\%) patients achieved CR of bone marrow after the first cycle of induction therapy to be enrolled in the DFS analysis and 86 (36.6\%) patients achieved MMR. Of the patients who achieve CR, 124(56.6\%) patients occurred recurrence. In the entire cohort, 57(24.3\%) patients received allo-HSCT. Finally, 75 died due to progressing disease or severe infection and 9 patients died for post-transplant complications.

Univariate and multivariable logistic regression analysis of patients' clinical parameters for the mutations in BCR-ABL kinase domain (KD)

To determine independent predictors for predicting the happen of BCR-ABL mutations, multivariate logistic regression was used for the three significant variables (higher WBC $(P=0.005)$, MMR after first induced chemotherapy $(P=0.053)$, and Age $\geq 45$ years $(P=0.072)$ identified by univariate analysis. Patients were categorized into two groups according to the median. Finally, higher WBC $\left(\geq 22 \times 10^{9} / \mathrm{L}\right.$; odds ratio $[O R]=2.127,95 \%$ confidence interval $[C I] 1.158-3.905 ; P=0.015)$ were significantly independent predictors of the mutations in BCR-ABL kinase domain in Ph + ALL. See Table 2 for details 
Table 2

Univariate and multivariable cox regression analysis of variables associated with $B C R-A B L$ kinase domain mutation in TKI-resistant patients with $\mathrm{Ph}+\mathrm{ALL}$

\begin{tabular}{|c|c|c|c|c|}
\hline \multirow[t]{3}{*}{ Variable } & \multicolumn{4}{|c|}{$\begin{array}{l}\text { BCR- ABL kinase domain mutation in patients with } \mathrm{Ph}+ \\
\text { ALL }\end{array}$} \\
\hline & \multicolumn{2}{|c|}{ Univariate analysis } & \multicolumn{2}{|c|}{ Multivariable analysis } \\
\hline & $\begin{array}{l}\text { Hazard ratio } \\
(95 \% \mathrm{Cl})\end{array}$ & $\begin{array}{l}\mathrm{P} \text { - } \\
\text { value }\end{array}$ & $\begin{array}{l}\text { Hazard ratio } \\
(95 \% \mathrm{Cl})\end{array}$ & $\begin{array}{l}\mathrm{P}- \\
\text { value }\end{array}$ \\
\hline Gender (male vs. female) & $\begin{array}{l}1.596(0.893- \\
2.850)\end{array}$ & 0.114 & & \\
\hline Age ( $\geq 45$ years vs. $₫ 45$ years) & $\begin{array}{l}1.700(0.853- \\
3.302)\end{array}$ & $0.072^{\star}$ & $\begin{array}{l}1.728(1.158- \\
3.905)\end{array}$ & 0.071 \\
\hline WBC ( $\geq 22$ vs. $\left.₫ 22 \times 10^{9} / \mathrm{L}\right)$ & $\begin{array}{l}2.337(1.291- \\
4.230)\end{array}$ & $0.005^{\star}$ & $\begin{array}{l}1.728(0.955- \\
3.126)\end{array}$ & $0.015^{*}$ \\
\hline $\mathrm{HB}(\geq 100 \mathrm{~g} / \mathrm{L}$ vs. $\otimes 100 \mathrm{~g} / \mathrm{L})$ & $\begin{array}{l}1.607(0.901- \\
2.867)\end{array}$ & 0.108 & & \\
\hline $\operatorname{PLT}\left(\geq 37 \times 10^{9} /\right.$ L vs. $\left.\otimes 37 \times 10^{9} / \mathrm{L}\right)$ & $\begin{array}{l}0.813(0.457- \\
1.445)\end{array}$ & 0.481 & & \\
\hline Blasts( $\geq 84 \%$ vs. $\varangle 84 \%)$ & $\begin{array}{l}0.843(0.471- \\
1.507)\end{array}$ & 0.564 & & \\
\hline $\begin{array}{l}\text { Relative BCR/ABL quantification ( } \geq \\
52 \% \text { vs. } \$ 52 \% \text { ) }\end{array}$ & $\begin{array}{l}0.823(0.453- \\
1.495)\end{array}$ & 0.523 & & \\
\hline $\begin{array}{l}\text { BCR/ABL transcript type (P210 vs. } \\
\text { P190) }\end{array}$ & $\begin{array}{l}0.755(0.417- \\
1.369)\end{array}$ & 0.355 & & \\
\hline $\begin{array}{l}\text { MMR after first induced chemotherapy } \\
\text { (Yes vs. No) }\end{array}$ & $\begin{array}{l}0.535(0.284- \\
1.007)\end{array}$ & $0.053^{*}$ & $\begin{array}{l}0.595(0.309- \\
1.146)\end{array}$ & 0.121 \\
\hline LDH $(\geq 620$ U/L vs. $₫ 620 U / L)$ & $\begin{array}{l}1.620(0.871- \\
3.013)\end{array}$ & 0.127 & & \\
\hline WT1 ( $\geq 0.5 \%$ vs. $\unrhd 0.5 \%)$ & $\begin{array}{l}0.836(0.371- \\
1.884)\end{array}$ & 0.665 & & \\
\hline IgH/TcR mutation (Yes vs. No) & $\begin{array}{l}1.169(0.505- \\
2.707)\end{array}$ & 0.716 & & \\
\hline ACAs (with ACAs vs. $\mathrm{Ph}+$ alone) & $\begin{array}{l}1.037(0.722- \\
1.488)\end{array}$ & 0.844 & & \\
\hline
\end{tabular}

\section{Comparison of treatment options in low-risk populations with BCR-ABL mutations}


Higher WBC $\geq 22 \times 109 / L$ was considered as independent predictors for BCR-ABL mutations. Among all the patients in our study, the OS and DFS in the cases with allo-HSCT was significantly longer than the cases without transplant $(P=0.000)$. Since WBC count, Age and MMR after the first course were significant predictors examined by univariate logistic regression analysis, we combined the three factors to explore the treatment options in low-risk populations with BCR-ABL mutations. We compared the OS and DFS between the transplant group and non-transplant group in subgroup according to the predictors. The advantage of transplantation therapy is not obvious as we can see in Fig. 2 in the defined low-risk subtype (with $\geq 2$ factors).

\section{$\mathrm{BCR} / \mathrm{ABL}$ gene mutations analysis}

Of patients with BCR-ABL mutation $(n=64)$, most patients $(n=57)$ received imatinib while only 7 received dasatinib as first-line therapy. The most frequent mutation detectable in $\mathrm{Ph}+\mathrm{ALL}$ treated with imatinib is T315I (detected in $54.7 \%$ of all the patients positive for mutations), followed by E255K(18.9\%)and then by Y253H (5.7\%). Twenty-eight patients included in our database were analyzed at the time of resistance to dasatinib (both after imatinib-resistance and used at initial). The Higher proportion of T315I and multiple mutations can be observed in dasatinib-resistance patients. Ponatinib was used in 8 patients up to December 2019. The use of nilotinib and flumatinib are occasional events. It's worth noting that 23 patients in our study get "compound" mutations during the sequential treatment by different TKIs. See

\section{Fig. 3 for details}

\section{Discussion}

The clinical course of patients with $\mathrm{Ph}+\mathrm{ALL}$ has dramatically improved with the current therapies combined with TKIs, including imatinib ${ }^{[10]}$, dasatinib ${ }^{[11]}$ and ponatinib ${ }^{[12]}$. Meanwhile, molecular remission has recently become a primary goal of treatment. MRD monitoring and BCR/ABL KD mutation are irreplaceable for the successful management of $\mathrm{Ph}+\mathrm{ALL}$ patients ${ }^{[2]}$. Nevertheless, $30 \%$ of the patients treated with imatinib relapse in a short time interval ${ }^{[13]}$ and there are still some obstructions that remain to be settled on the way to therapy optimization.

Fostered by the high genetic instability of ALL cells, patients with $\mathrm{Ph}+\mathrm{ALL}$ are highly prone to develop TKI-resistant mutations. The molecular mechanisms are poorly understood up to now and an in-depth evaluation is in need. BCR-ABL mutation has been implicated as a major cause of resistance to TKI therapy ${ }^{[5]}$, which emphasizes the utility and significance of BCR/ABL KD mutation screening analysis.

Clinically universal adjustment in TKI drugs is usually after the occurrence of resistance mutations, with a relatively high possibility of multiple mutations or sustained resistance. The sequential use of TKI could delay the selection of the best treatment. Therefore, the early prediction of BCR-ABL KD acquired mutations is essential. Up to now, few studies focused on the early prediction of acquired BCR/ABL mutation, especially for $\mathrm{Ph}+\mathrm{ALL}$. 
The most frequent detectable mutation in the Ph + ALL imatinib-resistant in our study is T315I (54.7\%), E225K(18.9\%) and $\mathrm{Y} 253 \mathrm{H}$ (5.7\%), which is consistent with the earlier reports ${ }^{[5,14]}$. Second-generation TKIs have increased therapeutic effect and have much fewer insensitive mutations ${ }^{[15,16]}$. However, sequential treatment by different TKIs may favor the accumulation of multiple mutations and the majority of dasatinib-resistant patients harbor "compound" mutations ${ }^{[17]}$. The T315I mutation is still a tough enemy for second-generation TKls and its proportion of dasatinib-resistant genetic alterations (57.1\%) has even increased in this research. It revealed the limitation of the efficacy of subsequent treatments. The selected choice of ponatinib and blinatumomab may bring benefits to relapsed $\mathrm{Ph}+\mathrm{ALL}$ patients.

Therefore, we retrospectively analyzed clinical, cytogenetic, hematological and molecular data collected from $235 \mathrm{Ph}+\mathrm{ALL}$ patients and identified high white blood cell count for predicting the ABL1 KD mutation. Previous studies show that high WBC count at diagnosis was a strong prognostic factor associated with a poor outcome in $\mathrm{Ph}+\mathrm{ALL}{ }^{[18,19]}$. Higher WBC count was considered as an independent risk of resistance to TKI in patients with $\mathrm{Ph}+\mathrm{ALL}$ and an arbitrary cutoff of $17 \times 109 \mathrm{WBCs} / \mathrm{L}$ was calculated in this research.

The allo-HSCT has long been demonstrated to be effective for Ph + ALL patients ${ }^{[20,21]}$. However, this approach has become controversial with the application of more potent TKIs, as well as more precise methods for monitoring MRD ${ }^{[22]}$. Daver et al. found that there was no survival advantage in patients aged 41 to 60 years receiving imatinib plus chemotherapy followed by allo-HSCT ${ }^{[23]}$. Raising attention towards a risk-modeled allotransplantation strategy could be predicted. Our results indicated that patients with a low-risk of BCR-ABL mutation could be candidates for TKI in combination with chemotherapy without the transplant. In other subgroups, allo-HSCT still confers superior survival for $\mathrm{Ph}+\mathrm{ALL}$ patients compared with pure TKIs combined chemotherapy. Nevertheless, it represents a tentative step of an attempt to "free- allo-HSCT approaches" in Ph + ALL.

The study has limitations due to its retrospective nature; Patients without achieving MMR after first induced chemotherapy are more prone to occur resistance mutation in univariate $(P=0.053)$ but not in multivariable logistic regression analysis $(P=0.121)$. In the future, larger-scale researches or an in-depth evaluation of molecular response need to be carried out.

\section{Conclusion}

Taken together, higher WBC $(P=0.015)$ are independent risks of resistance to TKI and raise the possibility of $\mathrm{KD}$ mutations in patients with $\mathrm{Ph}+\mathrm{ALL}$. There was no transplanting survival advantage in patients with the combination of at least two predictors of $B C R / A B L$ mutation indicating free-transplant could be considered in the patients with defined low-risk features.

\section{Declarations}


The authors alone are responsible for design and analysis of the study. There was no conflict of interest among authors.

\section{Author Contributions}

Rongrong Chen and Lulu Wang: major participation of the study, protocol development, analysis and interpretation of data; Lixia Zhu and Ting Shi: screening cases, collection of clinical variables, analysis and interpretation of data; Xiujin Ye: conception and design of the study and revision of the article.

\section{Ethics approval and consent to participate}

Our research was approved by The First Affiliated Hospital, College of Medicine, Zhejiang, University, Hangzhou, Zhejiang Province. The Ethical code is 2020 IIT833.

\section{Acknowledgements}

The research was supported by grants from Medical Health Science and Technology Project of Zhejiang Provincial Health Commission [No.2017KY059].

\section{Availability of data and material}

The data of this manuscript have been presented in the main paper.

\section{Competing interests}

The authors declare that they have no competing interests

\section{Consent for publication}

Not applicable

\section{Founding}

Not applicable

\section{References}

1. Wassmann B, Pfeifer H, Goekbuget N, Beelen DW, Beck J, Stelljes M, et al. Alternating versus concurrent schedules of imatinib and chemotherapy as front-line therapy for Philadelphia-positive acute lymphoblastic leukemia (Ph+ ALL). Blood. 2006;108(5):1469-77.doi:10.1182/blood-2005-114386

2. Soverini S, Bassan R, Lion T. Treatment and monitoring of Philadelphia chromosome-positive leukemia patients: recent advances and remaining challenges. Journal of hematology \& oncology. 2019;12(1):39.doi:10.1186/s13045-019-0729-2 
3. Yilmaz M, Kantarjian H, Ravandi-Kashani F, Short NJ, Jabbour E. Philadelphia chromosome-positive acute lymphoblastic leukemia in adults: current treatments and future perspectives. Clinical advances in hematology \& oncology : H\&O. 2018;16(3):216-23

4. Azam M, Seeliger MA, Gray NS, Kuriyan J, Daley GQ. Activation of tyrosine kinases by mutation of the gatekeeper threonine. Nature structural \& molecular biology. 2008;15(10):110918.doi:10.1038/nsmb.1486

5. Soverini S, De Benedittis C, Papayannidis C, Paolini S, Venturi C, lacobucci I, et al. Drug resistance and BCR-ABL kinase domain mutations in Philadelphia chromosome-positive acute lymphoblastic leukemia from the imatinib to the second-generation tyrosine kinase inhibitor era: The main changes are in the type of mutations, but not in the frequency of mutation involvement. Cancer. 2014;120(7):1002-9.doi:10.1002/cncr.28522

6. Arunachalam AK, Janet NB, Korula A, Lakshmi KM, Kulkarni UP, Aboobacker FN, et al. Prognostic value of MRD monitoring based on BCR-ABL1 copy numbers in Philadelphia chromosome positive acute lymphoblastic leukemia. Leukemia \& lymphoma. 2020:1-

8.doi:10.1080/10428194.2020.1811272

7. Soverini S, Mancini M, Bavaro L, Cavo M, Martinelli G. Chronic myeloid leukemia: the paradigm of targeting oncogenic tyrosine kinase signaling and counteracting resistance for successful cancer therapy. Molecular cancer. 2018;17(1):49.doi:10.1186/s12943-018-0780-6

8. Mahon FX, Réa D, Guilhot J, Guilhot F, Huguet F, Nicolini F, et al. Discontinuation of imatinib in patients with chronic myeloid leukaemia who have maintained complete molecular remission for at least 2 years: the prospective, multicentre Stop Imatinib (STIM) trial. The Lancet Oncology. 2010;11(11):1029-35.doi:10.1016/s1470-2045(10)70233-3

9. Pfeifer H, Wassmann B, Pavlova A, Wunderle L, Oldenburg J, Binckebanck A, et al. Kinase domain mutations of $B C R-A B L$ frequently precede imatinib-based therapy and give rise to relapse in patients with de novo Philadelphia-positive acute lymphoblastic leukemia (Ph+ ALL). Blood. 2007;110(2):72734.doi:10.1182/blood-2006-11-052373

10. Vignetti M, Fazi P, Cimino G, Martinelli G, Di Raimondo F, Ferrara F, et al. Imatinib plus steroids induces complete remissions and prolonged survival in elderly Philadelphia chromosome-positive patients with acute lymphoblastic leukemia without additional chemotherapy: results of the Gruppo Italiano Malattie Ematologiche dell'Adulto (GIMEMA) LAL0201-B protocol. Blood. 2007;109(9):36768.doi:10.1182/blood-2006-10-052746

11. Ottmann O, Dombret $H$, Martinelli G, Simonsson B, Guilhot F, Larson RA, et al. Dasatinib induces rapid hematologic and cytogenetic responses in adult patients with Philadelphia chromosome positive acute lymphoblastic leukemia with resistance or intolerance to imatinib: interim results of a phase 2 study. Blood. 2007;110(7):2309-15.doi:10.1182/blood-2007-02-073528

12. Cortes JE, Kim DW, Pinilla-Ibarz J, le Coutre PD, Paquette R, Chuah C, et al. Ponatinib efficacy and safety in Philadelphia chromosome-positive leukemia: final 5-year results of the phase 2 PACE trial. Blood. 2018;132(4):393-404.doi:10.1182/blood-2016-09-739086 
13. Zuo Z, Jones D, Yao H, Thomas DA, O'Brien S, Ravandi F, et al. A pathway-based gene signature correlates with therapeutic response in adult patients with Philadelphia chromosome-positive acute lymphoblastic leukemia. Modern pathology : an official journal of the United States and Canadian Academy of Pathology, Inc. 2010;23(11):1524-34.doi:10.1038/modpathol.2010.137

14. Hofmann WK, Jones LC, Lemp NA, de Vos S, Gschaidmeier H, Hoelzer D, et al. Ph(+) acute lymphoblastic leukemia resistant to the tyrosine kinase inhibitor STI571 has a unique BCR-ABL gene mutation. Blood. 2002;99(5):1860-2.doi:10.1182/blood.v99.5.1860

15. Kim DY, Joo YD, Lim SN, Kim SD, Lee JH, Lee JH, et al. Nilotinib combined with multiagent chemotherapy for newly diagnosed Philadelphia-positive acute lymphoblastic leukemia. Blood. 2015;126(6):746-56.doi:10.1182/blood-2015-03-636548

16. Foà R, Vitale A, Vignetti M, Meloni G, Guarini A, De Propris MS, et al. Dasatinib as first-line treatment for adult patients with Philadelphia chromosome-positive acute lymphoblastic leukemia. Blood. 2011;118(25):6521-8.doi:10.1182/blood-2011-05-351403

17. Shah NP, Skaggs BJ, Branford S, Hughes TP, Nicoll JM, Paquette RL, et al. Sequential ABL kinase inhibitor therapy selects for compound drug-resistant BCR-ABL mutations with altered oncogenic potency. The Journal of clinical investigation. 2007;117(9):2562-9.doi:10.1172/jci30890

18. Biondi A, Cario G, De Lorenzo P, Castor A, Conter V, Leoni V, et al. Long-term follow up of pediatric Philadelphia positive acute lymphoblastic leukemia treated with the EsPhALL2004 study: high white blood cell count at diagnosis is the strongest prognostic factor. Haematologica. 2019;104(1):e13e6.doi:10.3324/haematol.2018.199422

19. Rowe JM, Buck G, Burnett AK, Chopra R, Wiernik PH, Richards SM, et al. Induction therapy for adults with acute lymphoblastic leukemia: results of more than 1500 patients from the international ALL trial: MRC UKALL XII/ECOG E2993. Blood. 2005;106(12):3760-7.doi:10.1182/blood-2005-04-1623

20. Fielding AK, Rowe JM, Richards SM, Buck G, Moorman AV, Durrant IJ, et al. Prospective outcome data on 267 unselected adult patients with Philadelphia chromosome-positive acute lymphoblastic leukemia confirms superiority of allogeneic transplantation over chemotherapy in the pre-imatinib era: results from the International ALL Trial MRC UKALLXII/ECOG2993. Blood. 2009;113(19):448996.doi:10.1182/blood-2009-01-199380

21. Chalandon $Y$, Thomas $X$, Hayette $S$, Cayuela JM, Abbal C, Huguet F, et al. Randomized study of reduced-intensity chemotherapy combined with imatinib in adults with Ph-positive acute lymphoblastic leukemia. Blood. 2015;125(24):3711-9.doi:10.1182/blood-2015-02-627935

22. Litzow MR, Fielding AK, Luger SM, Paietta E, Ofran Y, Rowe JM, et al. The evolving role of chemotherapy and hematopoietic cell transplants in Ph-positive acute lymphoblastic leukemia in adults. Bone marrow transplantation. 2017;52(12):1592-8.doi:10.1038/bmt.2017.110

23. Daver N, Thomas D, Ravandi F, Cortes J, Garris R, Jabbour E, et al. Final report of a phase II study of imatinib mesylate with hyper-CVAD for the front-line treatment of adult patients with Philadelphia chromosome-positive acute lymphoblastic leukemia. Haematologica. 2015;100(5):65361.doi:10.3324/haematol.2014.118588

Page $12 / 15$ 


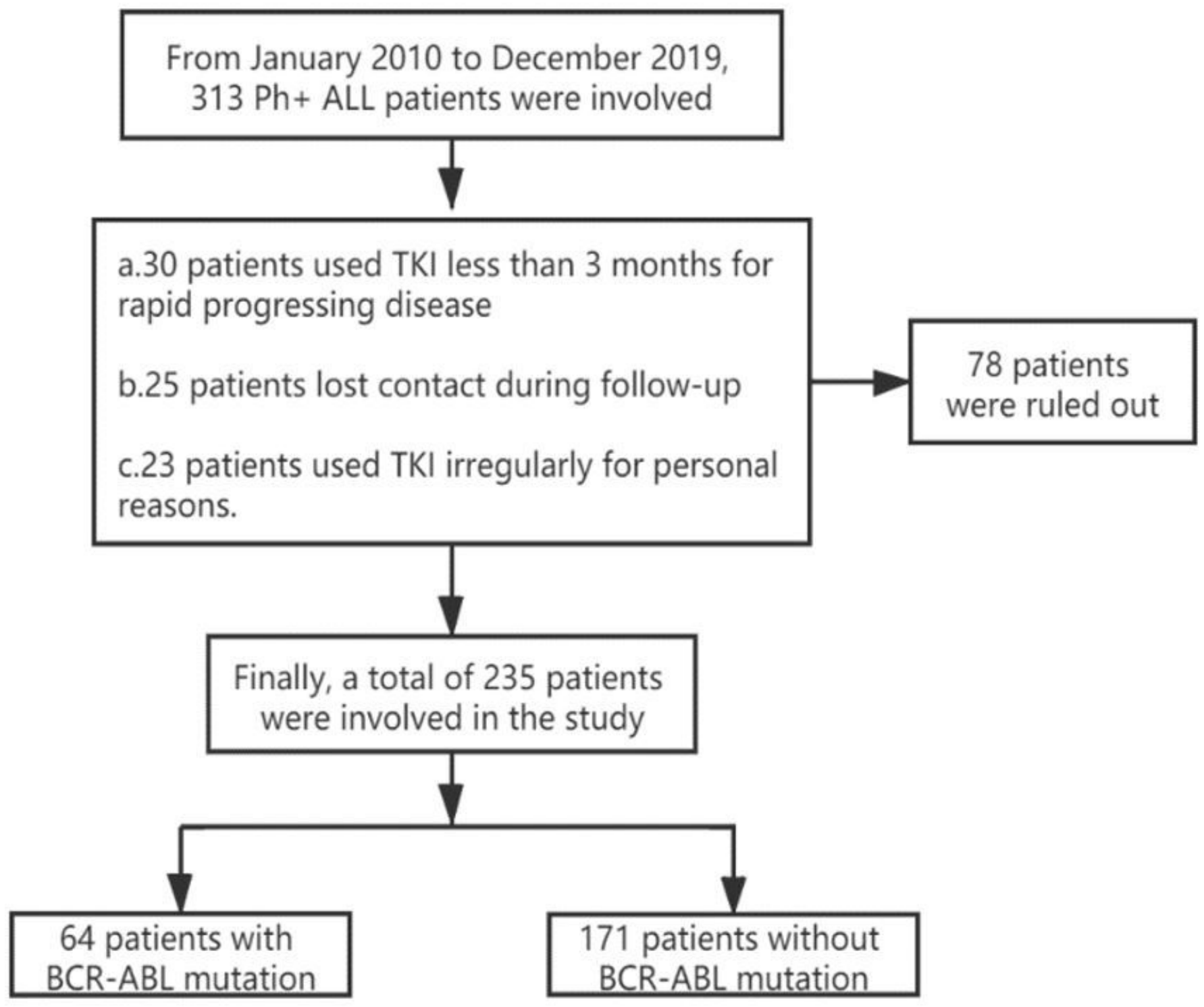

Figure 1

Flowchart of the patient enrollment process. 


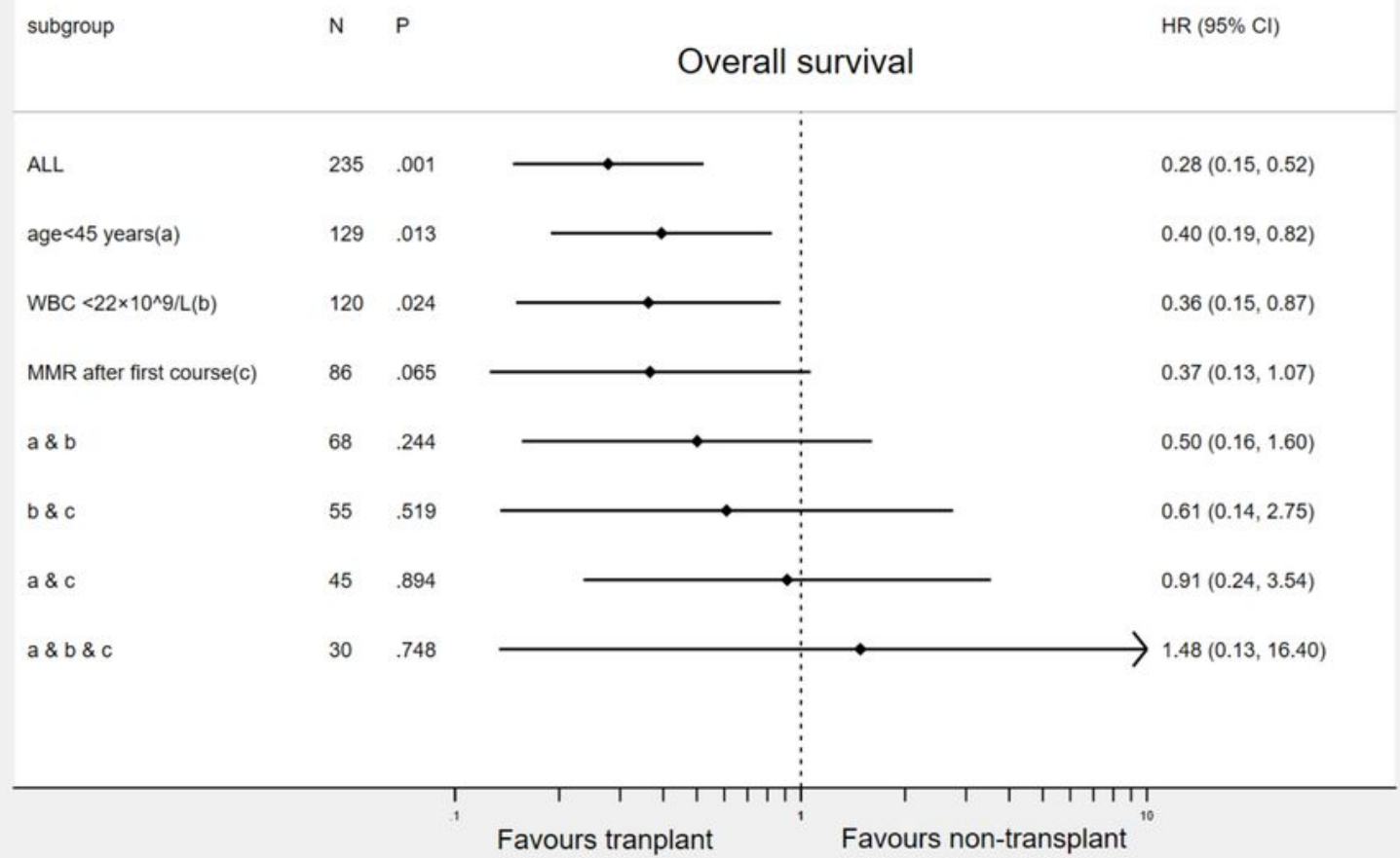

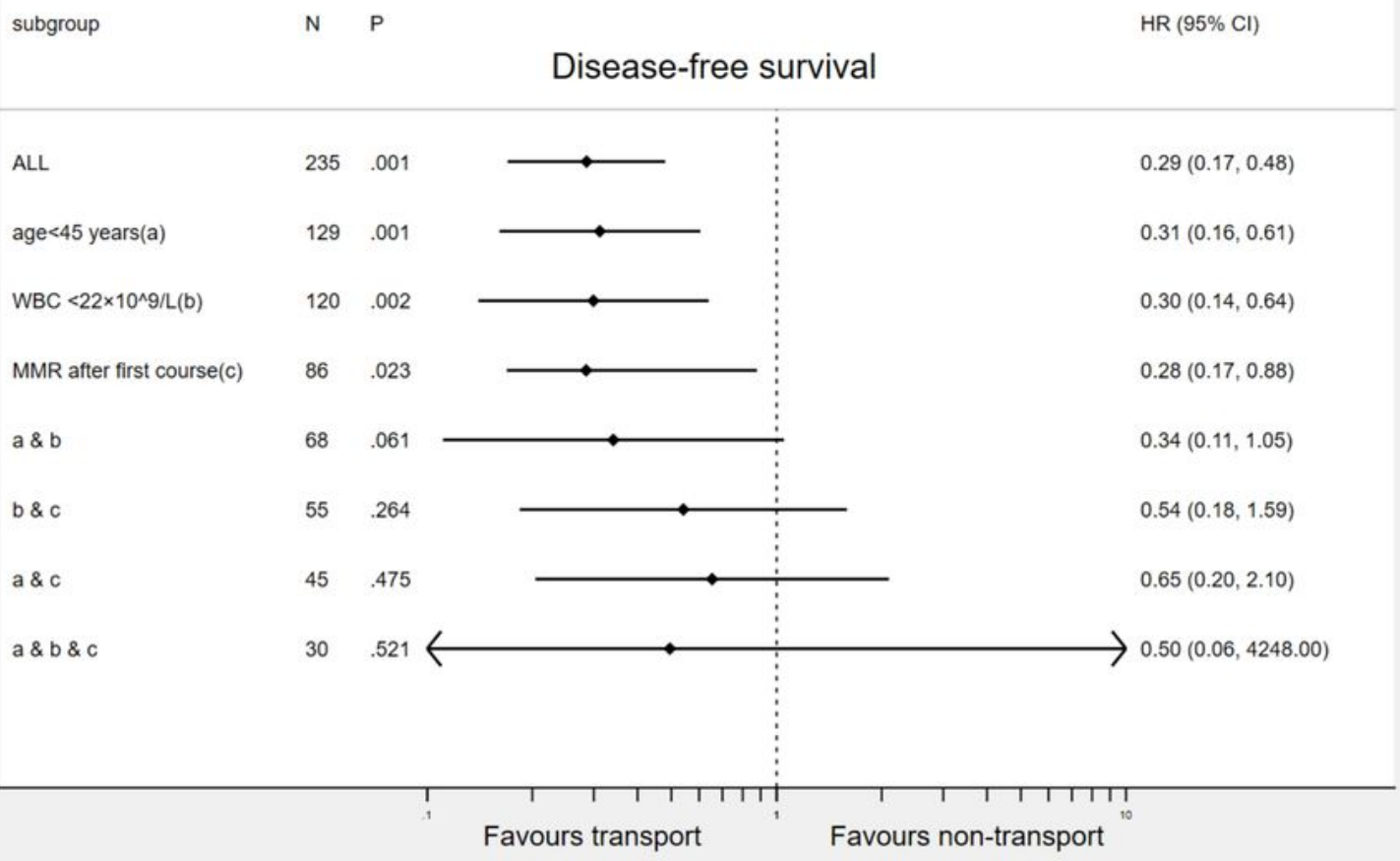

Figure 2

Compare OS and DFS between transplant group and non-transplant group in the in different risk populations 


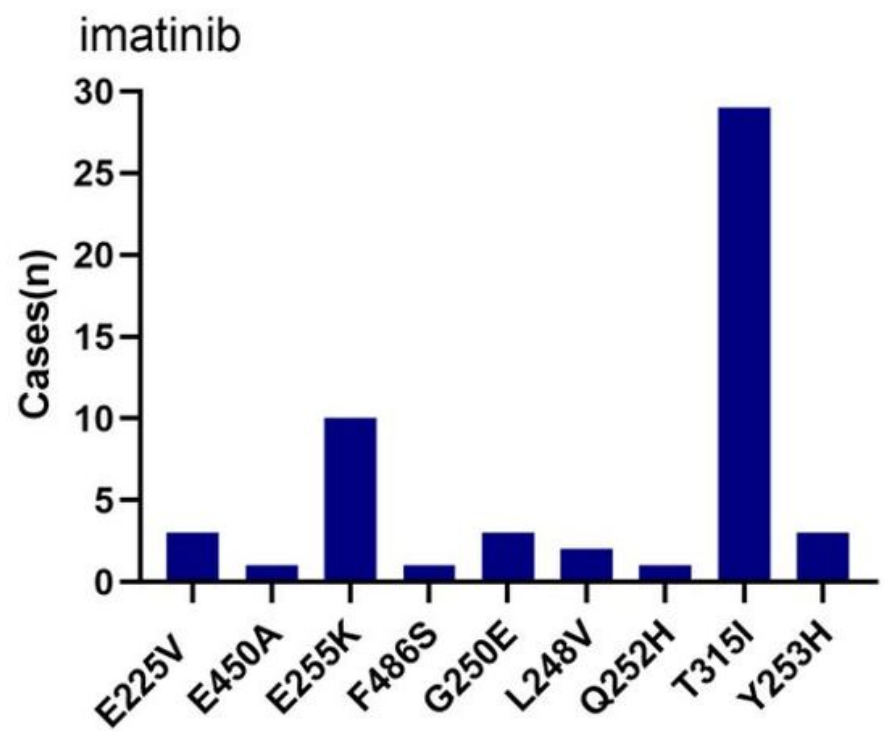

BCR-ABL mutation types

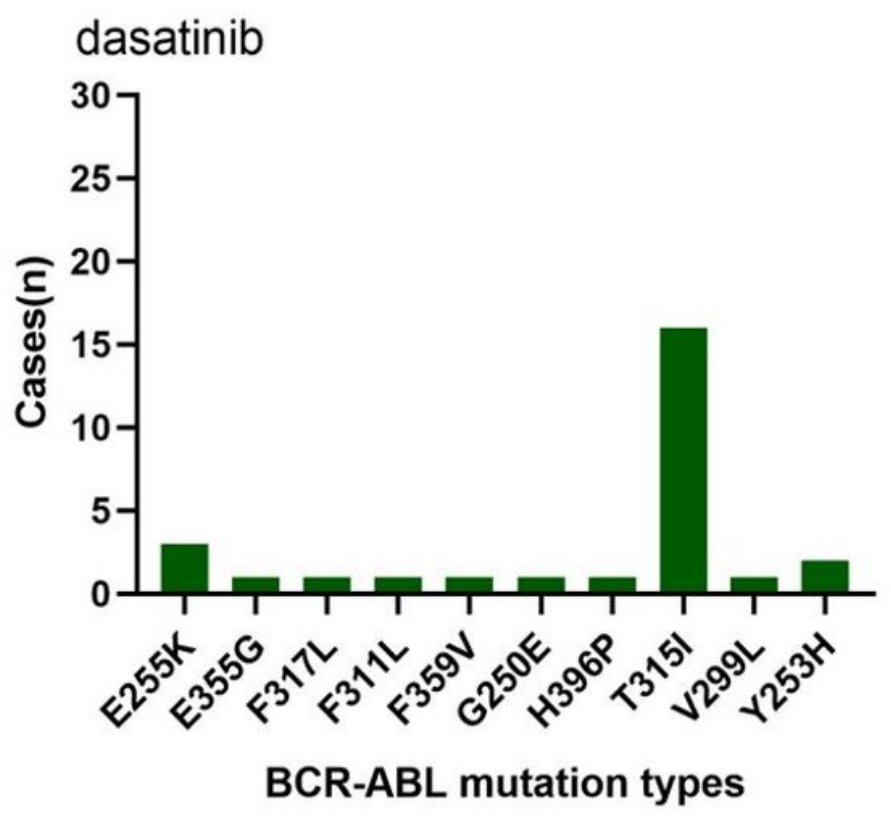

Figure 3

BCR-ABL KD mutations happens in the imatinib era and BCR-ABL KD mutations happen in the imatinib era (newly acquired) 